\title{
Intrarectal Lidocaine-Diltiazem-Meperidine Gel for Transrectal Ultrasound Guided Prostate Biopsy
}

\author{
Farsad Imani ${ }^{1}$; Yasaman Moghaddam ${ }^{1}$; Reza Shariat Moharari ${ }^{1}$; Farhad Etezadi ${ }^{1}$; \\ Mohammad Reza Khajavi ${ }^{1}$; Seyed Reza Hosseini ${ }^{2, *}$ \\ ${ }^{1}$ Department of Anesthesia, Sina Hospital, Tehran University of Medical Sciences, Tehran, Iran \\ ${ }^{2}$ Department of Urology, Sina Hospital, Tehran University of Medical Sciences, Tehran, Iran \\ *Corresponding author: Seyed Reza Hosseini, Department of Urology, Sina Hospital, Tehran University of Medical Sciences, Tehran, Iran. Tel: +98-9122083800, E-mail: srhoseini@tums.ac.ir
}

Received: August 6, 2014; Revised: September 1, 2014; Accepted: October 4, 2014

\begin{abstract}
Background:TRUS-guided needle biopsy of the prostate gland is the current standard method used for diagnosis of prostate cancer. Pain control during this procedure is through the use of i.v. sedation or local anaesthetic (LA), depending on clinician preference.

Objectives: The aim of this study was to evaluate the effectiveness of intrarectal lidocaine, lidocaine-diltiazem and lidocaine-meperidinediltiazem gel for anesthetizing transrectal ultrasound guided prostate biopsy.

Patients and Methods: In a randomized double-blind clinical trial, 100 consecutive patients were divided into three groups. The patients received one of the gels before transrectal ultrasound guided prostate needle biopsy: group A, intrarectal and perianal lidocaine, gel $1 \mathrm{~g}$; group B, intrarectal lidocaine gel, $1 \mathrm{~g}$, + perianal diltiazem, $1 \mathrm{~g}$; group C, intrarectal lidocaine gel, $1 \mathrm{~g},+$ meperidine, $25 \mathrm{mg}$, and perianal diltiazem, $1 \mathrm{~g}$. Visual analog pain scale was used to estimate pain during probe insertion and biopsy. Heart rate and blood pressure during probe insertion and biopsy were recorded too.

Results: The mean of visual analog pain scale was 4.5 in group A, 3.5 in group B, and 2.0 in group C during probe insertion $(\mathrm{P}$ value $=0.01)$. The mean of visual analog pain scale was 5.1 in group A, 3.5 group B, and 2.5 in group $C$ during biopsy ( $\mathrm{P}$ value $=0.001$ ). The groups were comparable for patients' age, weight, serum prostate-specific antigen (PSA), and prostate size (P>0.05). No side effects of meperidine and lidocaine including drowsiness, dizziness, tinnitus and light-headedness or requiring assistance for activity were noted.

Conclusions: Lidocaine-meperidine-diltiazem gel provides significantly better pain control than lidocaine-diltiazem gel and lidocaine gel alone during transrectal ultrasound guided prostate biopsy and probe insertion. This mixture gel is safe, easy to administer and well accepted by patients.
\end{abstract}

Keywords: Benign Prostatic Hyperplasia; Pain

\section{Background}

Transrectal ultrasound guided prostate biopsy has been the gold standard technique for early detection of prostate cancer (1), although identification of specific molecular markers for prostate cancer may lead to its earlier detection (2). As intravenous opioids have side effects (3), some authors have recommended periprostatic nerve block (4) and perianal-intrarectal lidocaine-prilocaine cream which provide better pain control than the two modalities alone for prostate biopsy anesthesia (5); but some have advised lidocaine $(4,6)$ or bupivacaine as a single agent $(7)$. These latter studies have suggested that rectal administration of lidocaine gel is a safe, simple modality, without any discomfort for patients (8). Not only local anesthetic agents but also intravenous anesthetic drugs (ketamine) (9) or opioids (10) that have local anesthetic effects have been used for local anesthetizing at urologic procedures.

In this study, we compared the effects of lidocaine gel alone, lidocaine gel plus diltiazem and lidocaine-meperidine-diltiazem in anesthetizing patients for transrectal ultrasound guided prostate biopsy. As calcium channel blockers such as diltiazem that cause relaxation of gastrointestinal smooth muscle have been shown to reduce resting anal sphincter pressure (11-13), we chose topical diltiazem to be added for groups B and C. Topical diltiazem has been an effective and safe treatment for anal fissure in people who have had failed topical glyceryl trinitrate $(11,12)$. As meperidine owns a local anesthetic property and can be absorbed to the mucosa $(14,15)$ of the rectum; we added this drug to group $\mathrm{C}$.

\section{Objectives}

The aim of this study was to evaluate the effectiveness of intrarectal lidocaine, lidocaine-diltiazem and lidocainemeperidine-diltiazem gel for anesthetizing transrectal ultrasound guided prostate biopsy.

\section{Patients and Methods}

After being approved by the Ethical Committee of Teh- 
ran University of Medical Sciences and registered in Iranian Registry of Clinical Trials (IRCT) center (number IRCT201211153773N7), this double-blinded randomized clinical trial was conducted on 100 patients awaiting transrectal ultrasound guided prostate biopsy in a teaching hospital from October 2012 to October 2013. Patients had the first experience from a prostate biopsy; it was considered that any previous painful experience from the procedure could have interfered with the present evaluation of pain. An informed written consent was obtained from the patients. Patients younger than 65 years of age, $>40$ $\mathrm{kg}$, with raised serum prostate-specific antigen (PSA), and abnormal digital rectal examination, were enrolled in this study. The exclusion criteria were coagulation disorders, hemorrhoidal diseases, severe cardiac or hepatic diseases, local anesthetic and opioids contraindication, and opioids addiction. Males were divided into three groups using a block random allocation process. Group A (38 patients) received lidocaine gel $2 \% 50 \mathrm{~mL}(1 \mathrm{~g})$ intrarectal and perianal, group B received lidocaine gel $2 \% 50 \mathrm{~mL}(1 \mathrm{~g})$ intrarectal and perianal plus diltiazem cream $1 \mathrm{~g}$ (36 patients); group $C$ received similar to group $B+$ meperidine $25 \mathrm{mg}$ which was added to the gel ( 26 patients). The mixing procedure of meperidine and diltiazem with lidocaine gel (group C): First, perianal diltiazem cream $1 \mathrm{~g}$ was applied in the form of application of diltiazem cream on and around the anus. Second, two lidocaine gel tubes were mixed by $25 \mathrm{mg}$ meperidine (prediluted in water and reached to $5 \mathrm{~mL}$ ) as follows: after pouring the first lidocaine gel tube into a 50$\mathrm{mL}$ syringe, meperidine was poured to the syringe. Then, the second lidocaine gel tube was added to the syringe. No diltiazem was added to the syringe containing meperidine and lidocaine gel. Therefore, no diltiazem was administered intrarectally; only diltiazem was applied on anus to relax its muscle. The gels were made by Sina Darou Company (Iran). The ultrasound probe was made by "Prosound" company (Japan). The mixture was administered 20-60 minutes before the biopsy. The drugs were administered by a physician; another physician who was unaware of the group allocation estimated the pain level. The patients were blinded to the composition of drugs. Visual analog pain scale was used to estimate the pain during probe insertion and biopsy. Heart rate and blood pressure during probe insertion and biopsy were recorded. Males were observed for effects of opioid (meperidine) or lidocaine overdose: drowsiness, dizziness, requiring assistance for normal activities or tinnitus and light-headedness. Males had 15-20 samples obtained at the time of biopsy.

For reducing the infection rate, all the males received a standard antibiotic prophylaxis: ofloxacin $300 \mathrm{mg}$ twice daily for three days, started eight hours before the procedure, although simple use of povidone-iodine for cleaning the rectum before transrectal ultrasound guided prostate biopsy has been reported to reduce the infection rate as well (16). The patients were asked to attempt to retain the gel. For comparing different main outcomes across the groups, we used one way analysis of variance. SPSS version 17 was used for statistical analysis. $P<5 \%$ was regarded significant.

\section{Results}

There was no statistical significance in prostate volume, weight, age, PSA levels and blood pressure between the three groups. Since the patients were asked to attempt to retain the gel, no patient expulsed it. The patients' characteristics are summarized in Table 1 . The mean systolic blood pressures during probe insertion were $151 \pm 26$ $\mathrm{mmHg}$ in group A, $151 \pm 17 \mathrm{mmHg}$ in group B and $153 \pm$ $20 \mathrm{mmHg}$ in group C, which were not significantly different $(\mathrm{P}$ value $=0.961)$. The mean systolic blood pressures during the biopsy were $147 \pm 24 \mathrm{mmHg}$ in group A, 145 $\pm 24 \mathrm{mmHg}$ in group B and $145 \pm 20 \mathrm{mmHg}$ in group C, which were not significantly different $(P$ value $=0.867$ ). The mean heart rates during probe insertion were $78 \pm 16$ beat/minute in group A, $79 \pm 14$ in group B and $83 \pm 15$ in group $C$, which were not significantly different $(\mathrm{P}$ value $=$ 0.651). The mean heart rates during biopsy were $84 \pm 14$ beat/minute in groups $A$ and $B$ and $70 \pm 16$ in group C, but they were not significantly different $(\mathrm{P}$ value $=0.01$ ).

The mean visual analog scale (VAS) during probe insertion were $4.5 \pm 1.6$ in group A, $3.5 \pm 1.7$ in group $B$ and $2 \pm 2.2$ in group $\mathrm{C}(\mathrm{P}$ value $=0.01)$. The mean VAS scores during the biopsy were $5.1 \pm 2.1$ in group $A, 3.5 \pm 2.3$ in group $B$ and $2.5 \pm 2.2$ in group $C$ ( $P$ value $=0.001$ ), which were significantly different. No side effects of opioids (meperidine) and lidocaine including drowsiness, dizziness, requiring assistance for activity along with tinnitus and lightheadedness from one hour before the procedure until two hours after were noted (overall three hours).

Table 1. Patients' Demographic Characteristics and Basal Hemodynamic Values ${ }^{a, b}$

\begin{tabular}{lccccc}
\hline Variable & Males & Group A & Group B & Group C & PValue \\
\hline Weight, $\mathbf{k g}$ & 71 & 72 & 72 & 70 & 0.764 \\
Age, $\mathbf{y}$ & 66 & 67 & 64 & 67 & 0.479 \\
Prostate volume, $\mathbf{m L}$ & 54 & 55 & 59 & 47 & 0.064 \\
PSA level, $\mathbf{n g} / \mathbf{m L}$ & 21 & 23 & 13 & 27 & 0.1 \\
Base heart rate & 77 & 76 & 77 & 78 & 0.891 \\
Base systolic blood pressure, $\mathbf{m m H g}$ & 146 & 151 & 144 & 143 & 0.136 \\
\hline
\end{tabular}

${ }^{a}$ Abbreviation: PSA, prostate specific antigen.

$\mathrm{b}$ Data are presented as mean. 


\section{Discussion}

Our data suggested that the lidocaine-meperidine-diltiazem mixture was significantly superior to the lidocainediltiazem gel and lidocaine gel alone for pain control during transrectal ultrasound guided prostate biopsy. Intrarectal local anesthetic gel is the easiest and most noninvasive method to reduce the transrectal biopsy pain. The results of previous studies have been ambiguous, and the volume, concentration and timing of administration have not been standardized (17). Pain during prostate biopsy has two components. Firstly, it originates from ultrasound probe insertion and secondly, it raises from the biopsy punctures. Luscombe and Cooke (18) reported that for $27 \%$ of males, probe insertion pain was as bad as or worse than the pain from needle biopsies. Several procedures are being undertaken routinely, but the use of anesthesia staff will obviously increase the cost. Therefore, providing a safe anesthesia without the use of anesthesia staff may be preferred in terms of financial matters. Some procedures, e.g. combination of perianalintrarectal lidocaine-prilocaine cream and periprostatic nerve block can be administered without the use of anesthesia staff, but injection discomfort and time assignment are the disadvantages. Transrectal gels may be administered easily and safely and it is a noninvasive procedure. As usual, males who are candidates for prostate biopsy are old and there is a clinically significant reduction in the intensity of pain perception with increasing age (19). Therefore, the use of only topical anesthesia for most males scheduled for prostate biopsy, especially the old ones, seems to be logical.

Cantiello (20) et al. has reported their study with Antrolin cream (trademark filed in Italy with the Ministry of Health) in addition to periprostatic nerve blocker with lidocaine and naropin. The cream was a mixture of $1.5 \%$ lidocaine and $0.2 \%$ nifedipine which are calcium channels blockers located along the anal sphincter fibro-muscular cell membranes, achieving muscle relaxation. The aim of cream administration was to provide pain control during probe insertion. The authors found that pain during probe insertion might be significantly reduced using Antrolin. We administered a calcium channels blocker (diltiazem) in groups B and C in purpose of lessening the perceived pain in comparison to lidocaine gel alone; the result was significant.

Limitations: lipid-soluble opioids such as fentanyl may be administered; they could have stronger analgesic effects, although the physician who is usually an urologist should learn more about the new lipid-soluble opioids; an instrument able to precisely dissolve opioid in gel is also required. Since these patients are rather old, an instrument measuring the pain i.e. Bispectral index (BIS) (or newly recognized as brain function assessment) or perfusion index (21) which represents a noninvasive measure of peripheral perfusion are suggested. The latter instrument has been used to determine proper manage- ment of pain, especially in patients unable to communicate their discomfort, although currently they can only be used in extremities. Measuring the plasma opioid (before and after opioids administration) assuring the opioid effect is another suggestion.

Lidocaine-meperidine-diltiazem mixture provides a significantly better pain control than lidocaine-diltiazem gel or lidocaine gel alone during transrectal ultrasound guided prostate biopsy. These intrarectal gels were safe, easy to administer, and well accepted by the patients.

\section{Acknowledgements}

The authors are indebted to the Research and Development Center of Sina Hospital (especially the epidemiologist colleague) for technical/statistical support.

\section{Authors' Contributions}

Design and study collection: Farsad Imani, Yasaman Moghaddam, Seyed Reza Hosseini. Management: Farsad Imani, Yasaman Moghaddam, Seyed Reza Hosseini. Analysis of the data: Farsad Imani, Seyed Reza Hosseini. Preparation, review, and approval of the manuscript: Reza Shariat Moharari, Farhad Etezadi, Mohammad Reza Khajavi, Farsad Imani, Yasaman Moghaddam, Seyed Reza Hosseini.

\section{References}

1. Hodge KK, McNeal JE, Terris MK, Stamey TA. Random systematic versus directed ultrasound guided transrectal core biopsies of the prostate.J Urol.1989;142(1):71-4.

2. Mazaris E, Tsiotras A. Molecular pathways in prostate cancer. Nephrourol Mon. 2013;5(3):792-800.

3. Mojtahedzadeh M, Hashemian F, Najafi A, Rouini M, Aghamir M, Tavakoli H, et al. Comparison of the analgesic profile and side effects of tramadol vs pethidine, following urologoical surgery. $J$ Pharm Sci. 2004;12(3):111-4.

4. Griwan MS, Kumar A, Sen J, Singh SK. Comparative evaluation of periprostatic nerve block and diclofenac patch in transrectal ultrasound-guided prostatic needle biopsy. Nephrourol Mon. 2012;4(3):560-4.

5. Giannarini G, Autorino R, Valent F, Mogorovich A, Manassero F, De Maria $\mathrm{M}$, et al. Combination of perianal-intrarectal lidocaineprilocaine cream and periprostatic nerve block for pain control during transrectal ultrasound guided prostate biopsy: a randomized, controlled trial. J Urol. 2009;181(2):585-91.

6. Seymour H, Perry MJ, Lee-Elliot C, Dundas D, Patel U. Pain after transrectal ultrasonography-guided prostate biopsy: the advantages of periprostatic local anaesthesia. BJU Int. 2001;88(6):540-4.

7. Rabets JC, Jones JS, Patel AR, Zippe CD. Bupivacaine provides rapid, effective periprostatic anaesthesia for transrectal prostate biopsy. BJU Int. 2004;93(9):1216-7.

8. Mallick S, Humbert M, Braud F, Fofana M, Blanchet P. Local anesthesia before transrectal ultrasound guided prostate biopsy: comparison of 2 methods in a prospective, randomized clinical trial.J Urol. 2004;171(2 Pt 1):730-3.

9. Moharari RS, Najafi A, Khajavi MR, Moharari GS, Nikoobakht MR. Intraurethral instillation of ketamine for male rigid cystoscopy. J Endourol. 2010;24(12):2033-6.

10. Khajavi M, Moharari R, Najafi A, Kazemi H, Ostovar A, Khashayar P. Intraurethral tramadol alleviates rigid cystoscopy - related pain in men. Int J Urol Nurs. 2013;7(3):133-7.

11. Griffin N, Acheson AG, Jonas M, Scholefield JH. The role of topical diltiazem in the treatment of chronic anal fissures that have failed glyceryl trinitrate therapy. Colorectal Dis. 2002;4(6):430-5. 
12. Carapeti EA, Kamm MA, Phillips RK. Topical diltiazem and bethanechol decrease anal sphincter pressure and heal anal fissures without side effects. Dis Colon Rectum. 2000;43(10):1359-62.

13. Amoli HA, Notash AY, Shahandashti FJ, Kenari AY, Ashraf H. A randomized, prospective, double-blind, placebo-controlled trial of the effect of topical diltiazem on posthaemorrhoidectomy pain. Colorectal Dis. 2011;13(3):328-32.

14. Jonnavithula N, Durga P, Kulkarni DK, Ramachandran G. Bilateral intra-oral, infra-orbital nerve block for postoperative analgesia following cleft lip repair in paediatric patients: comparison of bupivacaine vs bupivacaine-pethidine combination. Anaesthesia. 2007;62(6):581-5.

15. Brau ME, Koch ED, Vogel W, Hempelmann G. Tonic blocking action of meperidine on $\mathrm{Na}+$ and $\mathrm{K}+$ channels in amphibian peripheral nerves. Anesthesiology. 2000;92(1):147-55.

16. Ghafoori M, Shakiba M, Seifmanesh H, Hoseini K. Decrease in infection rate following use of povidone-iodine during transrectal ultrasound guided biopsy of the prostate: a double blind randomized clinical trial. Iran J Radiol. 2012;9(2):67-70.

17. Raber M, Scattoni V, Roscigno M, Rigatti P, Montorsi F. Perianal and intrarectal anaesthesia for transrectal biopsy of the prostate: a prospective randomized study comparing lidocaine-prilocaine cream and placebo. BJU Int. 2005;96(9):1264-7.

18. Luscombe CJ, Cooke PW. Pain during prostate biopsy. Lancet 2004;363(9424):1840-1.

19. Gibson SJ, Helme RD. Age-related differences in pain perception and report. Clin Geriatr Med. 2001;17(3):433-56.

20. Cantiello F, Imperatore V, Iannuzzo M, Scibelli G, Di Meo S, Fusco F, et al. Periprostatic nerve block (PNB) alone vs PNB combined with an anaesthetic-myorelaxant agent cream for prostate biopsy: a prospective, randomized double-arm study. BJUInt. 2009;103(9):1195-8.

21. Hagar H, Church S, Mandadi G, Pulley D, Kurz A. The perfusion index measured by a pulse oximeter indicates pain stimuli in anesthetized volunteers. Anesthesiol. 2004;101(514). 